\title{
Some remarks on general sum-connectivity coindex
}

\author{
M. Matejić, E. Milovanović, I. Milovanović
}

\begin{abstract}
Let $G=(V, E), V=\left\{v_{1}, v_{2}, \ldots, v_{n}\right\}$ be a simple connected graph with $n$ vertices, $m$ edges and a sequence of vertex degrees $d_{1} \geq d_{2} \geq \cdots \geq d_{n}>0, d_{i}=d\left(v_{i}\right)$. The general sumconnectivity coindex is defined as $\bar{H}_{\alpha}(G)=\sum_{i \nsim j}\left(d_{i}+d_{j}\right)^{\alpha}$, while multiplicative first Zagreb coindex is defined as $\bar{\Pi}_{1}(G)=\prod_{i \nsim j}\left(d_{i}+d_{j}\right)$. Here $\alpha$ is an arbitrary real number, and $i \nsim j$ denotes that vertices $i$ and $j$ are not adjacent. Some relations between $\bar{H}_{\alpha}(G)$ and $\bar{\Pi}_{1}(G)$ are obtained.
\end{abstract}

Keywords: Topological indices and coindices, sum-connectivity coindex, multiplicative Zagreb coindex.

\section{Introduction}

Let $G=(V, E), V=\left\{v_{1}, v_{2}, \ldots, v_{n}\right\}, E=\left\{e_{1}, e_{2}, \ldots, e_{m}\right\}$, be a simple connected graph with $n=|V|$ vertices and $m=|E|$ edges. With $d_{1} \geq d_{2} \geq \cdots \geq d_{n}>0, d_{i}=d\left(v_{i}\right)$, a sequence of vertex degrees of $G$ is designated. If vertices $v_{i}$ and $v_{j}$ are adjacent, we write $i \sim j$, otherwise we write $i \nsim j$. We define values $\bar{\Delta}_{e}$ and $\bar{\delta}_{e}$ as

$$
\bar{\Delta}_{e}=\max _{i \nsim j}\left\{d_{i}+d_{j}\right\} \quad \text { and } \quad \bar{\delta}_{e}=\min _{i \nsim j}\left\{d_{i}+d_{j}\right\} .
$$

A topological index of a graph is a numerical quantity which is invariant under automorphisms of the graph.

Two vertex-degree based topological indices, the first and the second Zagreb index, $M_{1}$ and $M_{2}$, are defined as $[7,8]$

$$
M_{1}=M_{1}(G)=\sum_{i=1}^{n} d_{i}^{2} \quad \text { and } \quad M_{2}=M_{2}(G)=\sum_{i \sim j} d_{i} d_{j}
$$

Manuscript received December 21, 2019; accepted February 14, 2020.

M. M. Matejić, E. I. Milovanović, I. Ž. Milovanović are with the Faculty of Electronic Engineering, Niš, Serbia 
As shown in [12], the first Zagreb index can be also expressed as

$$
M_{1}=\sum_{i \sim j}\left(d_{i}+d_{j}\right) .
$$

A so-called forgotten topological index, $F$, is defined as [6]

$$
F=F(G)=\sum_{i=1}^{n} d_{i}^{3}
$$

By analogy to $M_{1}$, the invariant $F$ can be written in the following way

$$
F=\sum_{i \sim j}\left(d_{i}^{2}+d_{j}^{2}\right)
$$

The general sum-connectivity index was conceived in [17] as

$$
H_{\alpha}(G)=\sum_{i \sim j}\left(d_{i}+d_{j}\right)^{\alpha},
$$

where $\alpha$ is an arbitrary real number. Some special cases of this index are the first Zagreb index $M_{1}(G)=H_{1}(G)$, the harmonic index $H(G)=2 H_{-1}(G)$ [5], the sum-connectivity index $S C(G)=H_{-1 / 2}(G)$ [18], and hyper-Zagreb index $H M(G)=H_{2}(G)$ [13]. It is not difficult to see that

$$
H M(G)=\sum_{i \sim j}\left(d_{i}+d_{j}\right)^{2}=F(G)+2 M_{2}(G) .
$$

In [4] a concept of coindices was introduced. In this case the sum runs over the edges of the complement of $G$. Thus, the first and the second Zagreb coindices are defined as [4]

$$
\bar{M}_{1}(G)=\sum_{i \nsim j}\left(d_{i}+d_{j}\right) \quad \text { and } \quad \bar{M}_{2}(G)=\sum_{i \nsim j} d_{i} d_{j},
$$

and the forgotten Zagreb coindex as [3] (see also [10]) as

$$
\bar{F}(G)=\sum_{i \nsim j}\left(d_{i}^{2}+d_{j}^{2}\right) .
$$

The general sum-connectivity coindex was defined in [14] as

$$
\bar{H}_{\alpha}(G)=\sum_{i \nsim j}\left(d_{i}+d_{j}\right)^{\alpha}
$$

where $\alpha$ is an arbitrary real number. Again, some special cases of $\bar{H}_{\alpha}(G)$ are apart from $\bar{M}_{1}(G)$, the sum-connectivity coindex $\overline{S C}(G)=\bar{H}_{-1 / 2}(G)$, the harmonic coindex $\bar{H}(G)=$ 
$2 \bar{H}_{-1}(G)$, the hyper Zagreb coindex $\overline{H M}(G)=\bar{H}_{2}(G)$ [15]. It is not difficult to see that the following identity holds

$$
\overline{H M}(G)=\bar{F}(G)+2 \bar{M}_{2}(G) .
$$

The multiplicative first Zagreb coindex was defined in [16] as

$$
\bar{\Pi}_{1}(G)=\prod_{i \nsim j}\left(d_{i}+d_{j}\right) .
$$

In this paper we determine the bound for the difference

$$
\bar{H}_{\alpha}(G)-\bar{m}\left(\bar{\Pi}_{1}(G)\right)^{\alpha / \bar{m}}
$$

where $\bar{m}=\frac{n(n-1)}{2}-m$.

\section{Preliminaries}

In this section we recall some analytical inequalities for the real number sequences that will be used in the subsequent considerations.

Let $a=\left(a_{i}\right)$ and $b=\left(b_{i}\right), i=1,2, \ldots, n$, be positive real number sequences with the properties

$$
0<r_{1} \leq a_{i} \leq R_{1}<+\infty \quad \text { and } \quad 0<r_{2} \leq b_{i} \leq R_{2}<+\infty .
$$

In [1] (see also [11]) the following inequality was proven

$$
\left|n \sum_{i=1}^{n} a_{i} b_{i}-\sum_{i=1}^{n} a_{i} \sum_{i=1}^{n} b_{i}\right| \leq n^{2} \gamma(n)\left(R_{1}-r_{1}\right)\left(R_{2}-r_{2}\right),
$$

where

$$
\gamma(n)=\frac{1}{n}\left\lfloor\frac{n}{2}\right\rfloor\left(1-\frac{1}{n}\left\lfloor\frac{n}{2}\right\rfloor\right)=\frac{1}{4}\left(1-\frac{(-1)^{n+1}+1}{2 n^{2}}\right) .
$$

Equality holds if and only if $R_{1}=a_{1}=\cdots=a_{n}=r_{1}$ or $R_{2}=b_{1}=\cdots=b_{n}=r_{2}$.

For the positive real number sequence $a=\left(a_{i}\right), i=1,2, \ldots, n$, the following inequality was proven in [9]

$$
\left(\sum_{i=1}^{n} \sqrt{a_{i}}\right)^{2} \leq(n-1) \sum_{i=1}^{n} a_{i}+n\left(\prod_{i=1}^{n} a_{i}\right)^{1 / n},
$$

with equality if and only if $a_{1}=a_{2}=\cdots=a_{n}$.

For the positive real number sequence $a=\left(a_{i}\right), i=1,2, \ldots, n$, with the property $a_{1} \geq$ $a_{2} \geq \cdots \geq a_{n}>0$, in [2] the following inequality was proven

$$
\sum_{i=1}^{n} a_{i}-n\left(\prod_{i=1}^{n} a_{i}\right)^{1 / n} \geq\left(\sqrt{a_{1}}-\sqrt{a_{n}}\right)^{2} .
$$

Equality holds if $a_{2}=a_{3}=\cdots=a_{n-1}=\sqrt{a_{1} a_{n}}$. 


\section{Main results}

In the next theorem we establish lower and upper bounds for the difference $\bar{H}_{\alpha}(G)-$ $\bar{m}\left(\bar{\Pi}_{1}(G)\right)^{\alpha / \bar{m}}$ depending on the parameters $\alpha, \bar{m}, \bar{\Delta}_{e}$ and $\bar{\delta}_{e}$.

Theorem 1. Let $G$ be a simple graph with $m \geq 2$ edges. If $\alpha \geq 0$, then

$$
\left(\bar{\Delta}_{e}^{\frac{\alpha}{2}}-\bar{\delta}_{e}^{\frac{\alpha}{2}}\right)^{2} \leq \bar{H}_{\alpha}(G)-\bar{m}\left(\bar{\Pi}_{1}(G)\right)^{\alpha / \bar{m}} \leq \bar{m}^{2} \gamma(\bar{m})\left(\bar{\Delta}_{e}^{\frac{\alpha}{2}}-\bar{\delta}_{e}^{\frac{\alpha}{2}}\right)^{2} .
$$

If $\alpha \leq 0, G \nsucceq K_{n}$, then

$$
\left(\bar{\delta}_{e}^{\frac{\alpha}{2}}-\bar{\Delta}_{e}^{\frac{\alpha}{2}}\right)^{2} \leq \bar{H}_{\alpha}(G)-\bar{m}\left(\bar{\Pi}_{1}(G)\right)^{\alpha / \bar{m}} \leq \bar{m}^{2} \gamma(\bar{m})\left(\bar{\delta}_{e}^{\frac{\alpha}{2}}-\bar{\Delta}_{e}^{\frac{\alpha}{2}}\right)^{2} .
$$

Equality on the left-hand side holds if $\alpha=0$, or $d_{i}+d_{j}=\sqrt{\bar{\Delta}_{e} \bar{\delta}_{e}}$, for any pair of nonadjacent vertices of $G$. Equality on the right-hand side holds if and only if $\alpha=0$ or $d_{i}+d_{j}$ is a constant for any pair of non adjacent vertices of $G$.

Proof. For $\alpha \geq 0, n:=\bar{m}, a_{i}=b_{i}:=\left(d_{i}+d_{j}\right)^{\frac{\alpha}{2}}, R_{1}=R_{2}=\bar{\Delta}_{e}^{\frac{\alpha}{2}}, r_{1}=r_{2}=\bar{\delta}^{\frac{\alpha}{2}}$, with summation performed over all non adjacent vertices of $G$, the inequality (1) becomes

$$
\bar{m} \sum_{i \nsim j}\left(d_{i}+d_{j}\right)^{\alpha}-\left(\sum_{i \nsim j}\left(d_{i}+d_{j}\right)^{\frac{\alpha}{2}}\right)^{2} \leq \bar{m}^{2} \gamma(\bar{m})\left(\bar{\Delta}_{e}^{\frac{\alpha}{2}}-\bar{\delta}_{e}^{\frac{\alpha}{2}}\right)^{2},
$$

that is

$$
\bar{m} \bar{H}_{\alpha}(G)-\left(\sum_{i \nsim j}\left(d_{i}+d_{j}\right)^{\frac{\alpha}{2}}\right)^{2} \leq \bar{m}^{2} \gamma(\bar{m})\left(\bar{\Delta}_{e}^{\frac{\alpha}{2}}-\bar{\delta}_{e}^{\frac{\alpha}{2}}\right)^{2} .
$$

For $\alpha \geq 0, n:=\bar{m}, a_{i}:=\left(d_{i}+d_{j}\right)^{\alpha}$, where summation is performed over all pairs of non adjacent vertices of $G$, the inequality (2) transforms into

$$
\left(\sum_{i \nsim j}\left(d_{i}+d_{j}\right)^{\frac{\alpha}{2}}\right)^{2} \leq(\bar{m}-1) \sum_{i \nsim j}\left(d_{i}+d_{j}\right)^{\alpha}+\bar{m}\left(\prod_{i \nsim j}\left(d_{i}+d_{j}\right)^{\alpha}\right)^{1 / \bar{m}},
$$

that is

$$
\left(\sum_{i \nsim j}\left(d_{i}+d_{j}\right)^{\frac{\alpha}{2}}\right)^{2} \leq(\bar{m}-1) \bar{H}_{\alpha}(G)+\bar{m}\left(\bar{\Pi}_{1}(G)\right)^{\alpha / \bar{m}} .
$$

Now from (5) and (6) we obtain right-hand side of (4). Equalities in (5) and (6), and consequently in the right-hand side of (4), hold if and only if $\alpha=0$ or $d_{i}+d_{j}$ is a constant for any pair of non adjacent vertices of $G$. 
For $\alpha \geq 0, n:=\bar{m}, a_{i}:=\left(d_{i}+d_{j}\right)^{\alpha}, a_{1}:=\bar{\Delta}_{e}^{\alpha}, a_{n}:=\bar{\delta}_{e}^{\alpha}$, with summation performed over all pairs of non adjacent vertices, the inequality (3) becomes

$$
\sum_{i \nsim j}\left(d_{i}+d_{j}\right)^{\alpha}-\bar{m}\left(\prod_{i \nsim j}\left(d_{i}+d_{j}\right)^{\alpha}\right)^{1 / \bar{m}} \geq\left(\bar{\Delta}_{e}^{\frac{\alpha}{2}}-\bar{\delta}_{e}^{\frac{\alpha}{2}}\right)^{2},
$$

from which left-hand part of (4) is obtained. Equality in (7), and consequently in (4), holds if $\alpha=0$ or $d_{i}+d_{j}=\sqrt{\bar{\Delta}_{e} \bar{\delta}_{e}}$ for any pair of non adjacent vertices of $G$.

The case $\alpha \leq 0$ is proved analogously, thus omitted.

Since for any $\bar{m}$ holds $\gamma(\bar{m}) \leq \frac{1}{4}$, we have the next corollary of Theorem 1 .

Corollary 1. Let $G$ be a simple graph with $m \geq 2$ edges. If $\alpha \geq 0$, then

$$
\bar{H}_{\alpha}(G)-\bar{m}\left(\bar{\Pi}_{1}(G)\right)^{\alpha / \bar{m}} \leq \frac{\bar{m}^{2}}{4}\left(\bar{\Delta}_{e}^{\frac{\alpha}{2}}-\bar{\delta}_{e}^{\frac{\alpha}{2}}\right)^{2} .
$$

If $\alpha \leq 0$ and $G \not K_{n}$, then

$$
\bar{H}_{\alpha}(G)-\bar{m}\left(\bar{\Pi}_{1}(G)\right)^{\alpha / \bar{m}} \leq \frac{\bar{m}^{2}}{4}\left(\bar{\delta}_{e}^{\frac{\alpha}{2}}-\bar{\Delta}_{e}^{\frac{\alpha}{2}}\right)^{2} .
$$

Equalities hold if and only if $\alpha=0$, or $d_{i}+d_{j}$ is a constant for any pair of non adjacent vertices of $G$.

For some specific values of parameter $\alpha$ the following inequalities are obtained.

Corollary 2. Let $G, G \nsupseteq K_{n}$, be a simple graph with $m \geq 2$ edges. Then we have

$$
\begin{aligned}
& \left(\frac{\sqrt{\bar{\Delta}_{e}}-\sqrt{\bar{\delta}_{e}}}{\sqrt{\bar{\Delta}_{e} \bar{\delta}_{e}}}\right)^{2} \leq \frac{1}{2} \bar{H}(G)-\bar{m}\left(\bar{\Pi}_{1}(G)\right)^{-1 / \bar{m}} \leq \bar{m}^{2} \gamma(\bar{m})\left(\frac{\sqrt{\bar{\Delta}_{e}}-\sqrt{\bar{\delta}_{e}}}{\sqrt{\bar{\Delta}_{e} \bar{\delta}_{e}}}\right)^{2} \leq \\
& \leq \frac{\bar{m}^{2}}{4}\left(\frac{\sqrt{\bar{\Delta}_{e}}-\sqrt{\bar{\delta}_{e}}}{\sqrt{\bar{\Delta}_{e} \bar{\delta}_{e}}}\right)^{2} \\
& \left(\frac{\sqrt[4]{\bar{\Delta}_{e}}-\sqrt[4]{\bar{\delta}_{e}}}{\sqrt[4]{\bar{\Delta}_{e} \bar{\delta}_{e}}}\right)^{2} \leq \overline{S C}(G)-\bar{m}\left(\bar{\Pi}_{1}(G)\right)^{-1 /(2 \bar{m})} \leq \bar{m}^{2} \gamma(\bar{m})\left(\frac{\sqrt[4]{\bar{\Delta}_{e}}-\sqrt[4]{\bar{\delta}_{e}}}{\sqrt[4]{\bar{\Delta}_{e} \bar{\delta}_{e}}}\right)^{2} \leq \\
& \leq \frac{\bar{m}^{2}}{4}\left(\frac{\sqrt[4]{\bar{\Delta}_{e}}-\sqrt[4]{\bar{\delta}_{e}}}{\sqrt[4]{\bar{\Delta}_{e} \bar{\delta}_{e}}}\right)^{2} \\
& \left(\sqrt{\bar{\Delta}_{e}}-\sqrt{\bar{\delta}_{e}}\right)^{2} \leq \bar{M}_{1}(G)-\bar{m}\left(\bar{\Pi}_{1}(G)\right)^{1 / \bar{m}} \leq \bar{m}^{2} \gamma(\bar{m})\left(\sqrt{\bar{\Delta}_{e}}-\sqrt{\bar{\delta}_{e}}\right)^{2} \leq \\
& \leq \frac{\bar{m}^{2}}{4}\left(\sqrt{\bar{\Delta}_{e}}-\sqrt{\bar{\delta}_{e}}\right)^{2}
\end{aligned}
$$




$$
\left(\bar{\Delta}_{e}-\bar{\delta}_{e}\right)^{2} \leq \overline{H M}(G)-\bar{m}\left(\bar{\Pi}_{1}(G)\right)^{2 / \bar{m}} \leq \bar{m}^{2} \gamma(\bar{m})\left(\bar{\Delta}_{e}-\bar{\delta}_{e}\right)^{2} \leq \frac{\bar{m}^{2}}{4}\left(\bar{\Delta}_{e}-\bar{\delta}_{e}\right)^{2}
$$

Equalities in the left-hand sides of the above inequalities hold if $d_{i}+d_{j}=\sqrt{\bar{\Delta}_{e} \overline{\boldsymbol{\delta}}_{e}}$ for any pair of non-adjacent vertices $v_{i}$ and $v_{j}$ of $G$. Equalities in the right-hand sides of the above inequalities hold if and only if $d_{i}+d_{j}$ is constant for any pair of non-adjacent vertices $v_{i}$ and $v_{j}$ of $G$.

Since $2 \bar{F}(G) \geq \overline{H M}(G)=\bar{F}(G)+2 \bar{M}_{2}(G) \geq 4 \bar{M}_{2}(G)$, the following is valid.

Corollary 3. Let $G$ be a simple graph with $m \geq 2$ edges. Then

$$
\begin{aligned}
4 \bar{M}_{2}(G)-\bar{m}\left(\bar{\Pi}_{1}(G)\right)^{2 / \bar{m}} & \leq \bar{m}^{2} \gamma(\bar{m})\left(\bar{\Delta}_{e}-\bar{\delta}_{e}\right)^{2} \leq \frac{\bar{m}^{2}}{4}\left(\bar{\Delta}_{e}-\bar{\delta}_{e}\right)^{2} \\
2 \bar{F}(G)-\bar{m}\left(\bar{\Pi}_{1}(G)\right)^{2 / \bar{m}} & \geq\left(\bar{\Delta}_{e}-\bar{\delta}_{e}\right)^{2} .
\end{aligned}
$$

Equalities hold if and only if $d_{i}=d_{j}$ for any pair of non adjacent vertices of $G$.

\section{References}

[1] M. Biernacki, H. Pidek, C. Ryll-Nardzewski, Sur une inequality des integralles, UNIV. MARIE CURIE-SKLODOWSKA, A4 (1950) 1-4.

[2] V. CirTOAJE, The best lower bound depended on two fixed variables for Jensen's inequality with order variables, J. INEQ. APPL. 2010 (2010)\# 12858.

[3] N. De, S. M. A. NAYEem, A. PAL, The F-coindex of some graph operations, SPRINGERPLUS, 5 (2016) ARTICLE 221.

[4] T. DošLIĆ, Vertex-weighted Wiener polynomials for composite graphs, ARS MATH. CONTEMP. 1 (2008) 66-80.

[5] S. Fajtlowicz, On conjectures of Graffiti-II, CONGr. Numer. 60 (1987) 187-197.

[6] B. Furtula, I. Gutman, A forgotten topological index, J. Math. Chem. 53 (2015) $1184-1190$.

[7] I. Gutman, N. Trinajstić, Graph theory and molecular orbitals. Total $\pi$-electron energy of alternant hydrocarbons, CHEM. PHYs. LETT. 17 (1972) 535-538.

[8] I. Gutman, B. Ruščić, N. Trinajstić, C. F. Wilcox, Graph theory and molecular orbitals. XII. Acyclic polyenes, J. CHEM. PHYs. 62 (1975) 3399-3405.

[9] H. Kober, On the arithmetic and geometric means and on Hölder's inequality, Proc. AMER. MATH. SOC. 9 (1958) 452-459.

[10] T. MANSOUR, C. SONG, The $a$ and $(a, b)$-analogs of Zagreb indices and coindices of graphs, INT. J. COMBINATRORICS 2012 (2012) ARTICLE ID909285.

[11] D. S. Mitrinović, P. M. Vasić, Analytic inequalities, Springer VerlaG, BerlinHEIDELBERG-NEW YORK, 1970. 
[12] S. Nikolić, G. Kovačević, A. Milićević, N. Trinajstić, The Zagreb indices 30 years after, CROAT. CHEM. ACTA 76 (2003) 113-124.

[13] G. H. ShIRdel, H. RezApour, A. M. SAYAD, The hyper-Zagreb index of graph operations, IRAN. J. MATH. ChEM. 4 (2013) 213-220.

[14] G. SU, L. XU, On the general sum-connectivity co-index of graphs, IRAN. J. MATH. CHEM. 2 (1) (2011) 89-98.

[15] M. VEYLAKI, M. J. NiKMEHR, The third and hyper-Zagreb coindex of some graph operations, J. APPL. MATH. COMPUT. 50 (2016) 315-325.

[16] K. XU, K. C. DAS, K. TANG, On the multiplicative Zagreb coindex of graphs, OpUSCULA MATH. 33 (1) (2013) 191-204.

[17] B. Zhou, N. Trinajstić, On general sum-connectivity index, J. MATH. ChEM. 47, (2010) 210-218.

[18] B. Zhou, N. Trinajstić, On a novel connectivity index, J. MATH. Chem. 46 (2009) $1252-1270$. 